\title{
DECAIMIENTO EXPONENCIAL DE LA SOLUCIÓN DÉBIL DE UNA ECUACIÓN DE ONDA NO LINEAL
}

\section{Yolanda Santiago Ayala ${ }^{1}$}

RESUMEN.- En este artículo demostramos que existe una única solución débil de una ecuación de onda no-lineal. Probamos la unicidad de solución utilizando el método de Visik - Ladyshenkaia. También, usando el Lema de diferencias de Nakao, probamos el decaimiento exponencial de la energía asociada al sistema.

PALABRAS CLAVE.- Existencia de solución. Método de Visik Ladyshenkaia. Lema de Nakao. Decaimiento exponencial.

\section{EXPONENTIAL DECAY OF THE WEAK SOLUTION OF A NONLINEAR WAVE EQUATION}

\begin{abstract}
In this article, we prove the existence and uniqueness of the weak solution of a nonlinear wave equation. We prove the uniqueness by using the Visik - Ladyshenkaia Method. Also, using the Nakao's Lemma, we prove the exponential decay of the energy associated to the system.
\end{abstract}

KEYWORDS.- Existence of solution. Visik-Ladyshenkaia Method. Nakao's Lemma. Exponential decay.

\section{INTRODUCCIÓN}

Estudiamos la siguiente ecuación de onda no lineal,

$$
\begin{aligned}
& u_{t t}-\Delta u+u^{3}+u_{t}=0 \text { en } \Omega \times(0,+\infty) \\
& u=0 \text { en } \partial \Omega \times \mathbb{R}^{+} \\
& u(x, 0)=u_{0}(x), u_{t}(x, 0)=u_{1}(x),
\end{aligned}
$$

donde $\Omega \subset \mathbb{R}^{3}$, es abierto y acotado con frontera $\partial \Omega$ regular.

El problema (1.1) - (1.3) también puede ser visto como la ecuación de evolución de primer orden:

$$
\mid \begin{aligned}
& U_{t}=A U+N U \\
& U(0)=U_{0}
\end{aligned} \quad \text { donde } A=\left(\begin{array}{ll}
0 & I \\
\Delta & 0
\end{array}\right) \text { y } \quad N\left(\begin{array}{l}
u \\
v
\end{array}\right)=\left(\begin{array}{c}
0 \\
-u^{3}-v
\end{array}\right)
$$

\footnotetext{
' Universidad Nacional Mayor de San Marcos. Facultad de Ciencias Matemáticas. e-mail: ysantiagoa@unmsm.edu.pe
} 
Si pretendemos usar técnicas de Semigrupos [9], sería difícil acotar $v^{2}+v u+u^{2}$. De ahí que en este artículo demostraremos la existencia de solución débil del problema (1.1) - (1.3), vía el método de Faedo - Galerkin. Para probar la unicidad de solución utilizaremos el método de Visik - Ladyshenkaia. Para ver la propiedad disipativa del sistema, multiplicamos la ecuación (1.1) por $u_{t}$ y obtenemos

$$
\frac{\partial E}{\partial t}(t)=-\int_{\Omega}\left|u_{t}(t)\right|^{2} d x
$$

donde

$$
E(t)=\frac{1}{2} \int_{\Omega}\left\{\left|u_{t}(t)\right|^{2}+|\nabla u(t)|^{2}\right\} d x+\frac{1}{4} \int_{\Omega} u^{4}(t) d x
$$

es la energía asociada al sistema (1.1) - (1.3). De la igualdad (1.4) tenemos que $E^{\prime}(t)$ es no positiva, es decir la energía es acotada y decreciente, pero no se conoce que sucede con $E(t)$ cuando el tiempo va para infinito. Usando el Lema de diferencias de Nakao probaremos que la energía decae exponencialmente a cero, cuando $t \rightarrow+\infty$.

Todo esto lo resumimos en nuestro resultado principal.

Teorema 1.1 (Resultado principal) Sean $u_{0} \in H_{0}^{1}(\Omega) \cap L^{4}(\Omega)$ y $u_{1} \in L^{2}(\Omega)$. Entonces el Problema (1.1) - (1.3) tiene una única solución débil que decae exponencialmente a cero cuando el tiempo va para infinito; esto es existen constantes positivas $C$ y $\gamma$ de modo que se satisface

$$
E(t) \leq E(0) C e^{-\gamma t}, \forall t \geq 1 \text {. }
$$

\section{PRELIMINARES}

Lema 2.1 (Desigualdad de Gronwall) Sea f continua y no negativa en $[0, a]$ y $K$ una constante. Si se satisface:

$$
f(t) \leq K+\int_{0}^{t} f(s) d s, \forall t \in(0, a]
$$

entonces $f(t) \leq K e^{t}, \forall t \in(0, a]$.

En particular: si $K=0$ entonces $f(t) \equiv 0$.

Lema 2.2 En $U$ un abierto acotado de $\mathbb{R}_{x}^{n} \times \mathbb{R}_{t}$, sean $g_{\mu}$ y $g$ funciones de $L^{s}(U)$ con $1<s<+\infty,\left|g_{\mu}\right|_{L^{s}(U)} \leq C \quad y \quad g_{\mu} \rightarrow g$ en c. $t$. p. en U. Entonces $g_{\mu} \rightarrow g$ débilmente en $L^{s}(U)$

Prueba.- Sea $N \in \mathbb{N}, E_{N}:=\left\{x, x \in U\right.$ tal que $\left.\left|g_{\mu}(x)-g(x)\right| \leq 1, \mu \geq N\right\}$. Los conjuntos $E_{N}$ son 
conjuntos medibles, crecientes sobre $N$ y $m\left(E_{N}\right) \rightarrow m(U)$ cuando $N \rightarrow+\infty$, donde $m(A)$ es la medida de $A$.

Así, definimos $\phi_{N}:=\left\{\psi \in L^{r}(U)\right.$ tal que $\frac{1}{r}+\frac{1}{s}=1$ y soporte de $\left.\psi \subset E_{N}\right\}$, y denotamos por $\phi_{N}:=\bigcup_{N=1}^{\infty} \phi_{N}$, esto es $\phi$ es denso en $L^{r}(U)$

Sea $\psi \in \phi$ entonces existe $N_{0}$ tal que $\psi \in \phi_{N_{0}}$. Tomando $\mu \geq N_{0}$ tenemos que $\left|\psi\left(g_{\mu}-g\right)\right| \leq|\psi| \rightarrow 0$ c.t.p. Luego el Teorema de Lebesgue nos garantiza que $\int_{U} \psi\left(g_{\mu}-g\right) d x \rightarrow 0$ cuando $\mu \rightarrow+\infty$.

Así hemos probado que

$$
\text { si } \psi \in \phi \Rightarrow \int_{U} \psi\left(g_{\mu}-g\right) d x \rightarrow 0 \text { cuando } \mu \rightarrow+\infty \text {. }
$$

Desde que $\phi$ es denso en $L^{r}(U)$, entonces (2.1) prueba el Lema:

Lema 2.3 Sea $f \in L^{p}(0, T ; X), f_{t} \in L^{p}(0, T ; X)$ con $1 \leq p \leq+\infty$. Entonces existe una función continua $g$ en $[0, T]$ tal que $g=f$ en casi todo punto de $X$.

Prueba.- Ver [4].

Lema 2.4 Sea $H$ un espacio de Hilbert separable, $V \subset H$ una inclusión continua con $V$ denso en $H, 1 \leq p \leq \infty$. Si $f \in L^{p}(0, T ; V)$ y $f_{t} \in L^{p}(0, T ; H)$ entonces $f \in C([0, T], H)$. Por otro lado si $p=\infty$. entonces $f \in C_{\text {débilmente }}([0, T] ; V)$ (f es débilmente continua en $\left.[0, T]\right)$, i.e. $\forall g \in V^{*},<f(t), g>_{V V^{*}} \rightarrow<f\left(t_{0}\right), g>_{V V^{*}}$ cuando $t \rightarrow t_{0}, \forall t_{0} \in[0, T]$.

Prueba.- Ver [5].

Teorema 2.1 (Desigualdad de Poincaré) Sea $\Omega \subset \mathbb{R}^{n}$ un dominio acotado en una dirección, entonces existe una constante $C_{p}>0$ tal que $|u|_{L^{2}} \leq C_{p}|\nabla u|_{L^{2}}, \forall u \in H_{0}^{1}(\Omega)$. La constante $C_{p}$ es denominada la constante de Poincaré.

Prueba.- Ver [2].

Teorema 2.2 (Rellich - Kondrachoff) Sea $Q=(0, T) \times \Omega$, asi $H^{1}(Q) \mapsto L^{2}(Q)$ es una inyección compacta.

Prueba.- Ver [1]. 
Teorema 2.3 (Dunford - Pettis) $L^{\infty}\left(0, T ; H_{0}^{1}(\Omega) \cap L^{4}(\Omega)\right.$ ) (resp. $L^{\infty}\left(0, T ; L^{2}(\Omega)\right.$ ) es el dual de $L^{1}\left(0, T ; H^{-1}(\Omega)+L^{\frac{4}{3}}(\Omega)\right) \quad\left(\right.$ resp. $L^{1}\left(0, T ; L^{2}(\Omega)\right)$.

Prueba.- Ver [4].

Teorema 2.4 (Inmersión de Sobolev) Sea $\Omega=\mathbb{R}^{n}$ ó un abierto acotado con frontera de clase $C^{1} \dot{o} \mathbb{R}_{+}^{n}, m \in \mathbb{N}, 1 \leq p<+\infty$. Si $R=\frac{1}{p}-\frac{m}{n}$ entonces valen los siguientes enunciados con inclusiones continuas:

1. Si $R>0$ entonces $W^{m, p}(\Omega) \subset L^{q}(\Omega)$, donde $q=\frac{1}{R}$.

2. Si $R>0$ entonces $W^{m, p}(\Omega) \subset L^{q}(\Omega)$, donde $q \in[p,+\infty)$.

3. Si $R>0$ entonces $W^{m, p}(\Omega) \subset L^{\infty}(\Omega)$.

Prueba.- Ver [1], [2].

Lema 2.5 (Lema de diferencias de Nakao) Sea $\phi: \mathbb{R}^{+} \rightarrow \mathbb{R}^{+}$una función acotada con $\phi(0)>0$ y supongamos que existe $M>0$ tal que

$$
\sup _{s \in[t, t+1]} \phi(s) \leq M\{\phi(t)-\phi(t+1)\}, \forall t \geq 1
$$

Entonces existen $A>0$ y $M_{1}>0$ tal que $\phi(t) \leq M_{1} e^{-A t}, \forall t \geq 1$.

Esto quiere decir que " $\phi(t)$ decae exponencialmente a cero cuando $t \rightarrow+\infty$ ".

Prueba.- En [11] se presenta en detalle la demostración del Lema y otras aplicaciones. Es importante citar los trabajos de Nakao [6], [7] y [8].

\section{EXISTENCIA Y UNICIDAD}

\section{Prueba del Teorema 1.1}

\section{Prueba de la existencia}

Usaremos el método de Galerkin. Desde que $H_{0}^{1}(\Omega) \cap L^{4}(\Omega)$ es separable, tenemos que existe $\left\{w_{i}\right\}_{i \in \mathbb{N}}$ un subconjunto numerable y denso en $H_{0}^{1}(\Omega) \cap L^{4}(\Omega)$. i.e. $\forall m \in \mathbb{N}, w_{1}, \ldots, w_{m}$ son linealmente independientes y las combinaciones lineales finitas de $w_{i}$ son densas en $H_{0}^{1}(\Omega) \cap L^{4}(\Omega)$.

Denotemos por $u_{m}=u_{m}(t)$ a las soluciones aproximadas para nuestro problema, siendo $u_{m}(t)=\sum_{i=1}^{m} g_{i m}(t) w_{i}$, son determinados por las condiciones: 


$$
\begin{aligned}
0= & <u_{m}^{\prime \prime}(t), w_{j}>+a\left(u_{m}(t), w_{j}\right)+<u_{m}^{3}(t), w_{j}> \\
& +<u_{m}^{\prime}(t), w_{j}>, i \leq j \leq m .
\end{aligned}
$$

donde $a(u, v)=\sum_{i=1}^{3} \int_{\Omega} \frac{\partial u}{\partial x_{i}} \frac{\partial v}{\partial x_{i}} d x$.

$$
\begin{aligned}
& u_{m}(0)=u_{o m}, \quad u_{o m}=\sum_{i=1}^{m} \alpha_{i m} w_{i} \rightarrow_{m \rightarrow+\infty} u_{0} \text { en } H_{0}^{1}(\Omega) \cap L^{4}(\Omega), \\
& u_{m}^{\prime}(0)=u_{1 m}, \quad u_{1 m}=\sum_{i=1}^{m} \beta_{i m} w_{i} \rightarrow_{m \rightarrow+\infty} u_{1} \text { en } L^{2}(\Omega) .
\end{aligned}
$$

El sistema de ecuaciones diferenciales ordinarias no lineales (3.1) con las condiciones iniciales (3.2) - (3.3) tiene solución, desde que $\operatorname{det}\left(w_{i}, w_{j}\right) \neq 0$ ya que $\left\{w_{1}, \ldots w_{m}\right\}$ son linealmente independientes. Así existen soluciones $u_{m}$ en $\left[0, t_{m}\right]$.

Afirmamos que $t_{m}=T$ (Estimaciones apriori).

Multiplicando la ecuación (3.1) por $g_{i m}^{\prime}(t)$ y sumando en $i$, tenemos

$$
\begin{aligned}
0= & <u_{m}^{\prime \prime}(t), u_{m}^{\prime}(t)>+a\left(u_{m}(t), u_{m}^{\prime}(t)\right)+<u_{m}^{3}(t), u_{m}^{\prime}(t)> \\
& +<u_{m}^{\prime}(t), u_{m}^{\prime}(t)>, \\
= & \frac{1}{2} \frac{\partial}{\partial t}\left\{\left|u_{m}^{\prime}(t)\right|^{2}+a\left(u_{m}(t), u_{m}(t)\right)\right\}+\frac{1}{4} \frac{\partial}{\partial t}\left\{\int_{\Omega} u_{m}^{4}(t) d x\right\} \\
& +\int_{\Omega}\left|u_{m}^{\prime}(t)\right|^{2} d x .
\end{aligned}
$$

Defina,

$$
\|v\|:=\sqrt{a(v, v)}
$$

$\|$. $\|$ es una norma en $H_{0}^{1}(\Omega)$ y debido a desigualdad de Poincaré Teorema 2.1 se tiene que es equivalente a $|\cdot|_{H^{1}(\Omega)}$.

Integrando de 0 a $t$ la igualdad (3.4), tenemos

$$
\begin{aligned}
& \frac{1}{2}\left\{\left|u_{m}^{\prime}(t)\right|_{L^{2}(\Omega)}^{2}+\left\|u_{m}(t)\right\|_{H_{0}^{2}(\Omega)}^{2}\right\}+\frac{1}{4}\left|u_{m}(t)\right|_{L^{4}(\Omega)}^{4}+\int_{0}^{t}\left|u_{m}^{\prime}(t)\right|_{L^{2}(\Omega)}^{2} d s \\
& =\frac{1}{2}\{\underbrace{\left|u_{m}^{\prime}(0)\right|^{2}}_{=u_{i m}}+\left\|u_{m}(0)\right\|^{2}\}+\left.\frac{1}{4} \underbrace{\left|u_{m}(0)\right|}_{=u_{o m}}\right|_{L^{4}(\Omega)} ^{4} \\
& \rightarrow_{m \rightarrow+\infty} \frac{1}{2}\left\{\left|u_{1}\right|^{2}+\left\|u_{0}\right\|^{2}\right\}+\left|u_{0}\right|_{L^{4}(\Omega)}^{4}
\end{aligned}
$$


Luego,

$$
\frac{1}{2}\left\{\left|u_{m}^{\prime}(t)\right|_{L^{2}(\Omega)}^{2}+\left\|u_{m}(t)\right\|_{H_{0}^{2}(\Omega)}^{2}\right\}+\frac{1}{4}\left|u_{m}(t)\right|_{L^{4}(\Omega)}^{4}+\int_{0}^{t}\left|u_{m}^{\prime}(t)\right|_{L^{2}(\Omega)}^{2} d s \leq C
$$

donde $C$ es independiente de $m$.

También, $\left|u_{m}^{\prime}(t)\right|_{L^{2}(\Omega)}^{2} \leq 2 C \leq 2 C+\int_{0}^{t}\left|u_{m}^{\prime}(t)\right|_{L^{2}(\Omega)}^{2} d s$. Usando la desigualdad de Gronwall Lema

2.1, tenemos $\left|u_{m}^{\prime}(t)\right|_{L^{2}(\Omega)}^{2} \leq 2 C e^{t}$, donde $C$ es independiente de $m$.

Por otro lado, de (3.5) tenemos

$$
\left\|u_{m}(t)\right\|_{H_{0}^{1}(\Omega)}^{2}+\left\|u_{m}(t)\right\|_{L^{4}(\Omega)}^{4} \leq 4 C .
$$

De ahí que

$$
\left\|u_{m}(t)\right\|_{H_{0}^{1}(\Omega)}+\left\|u_{m}(t)\right\|_{L^{4}(\Omega)} \leq \widehat{C}
$$

donde $\hat{C}$ es independiente de $m$.

Luego $t_{m}=T$.

Por otro lado cuando $m \rightarrow+\infty$, tenemos que

$$
\begin{aligned}
& u_{m} \text { cae en un conjunto acotado de } L^{\infty}\left(0, T ; H_{0}^{1}(\Omega) \cap L^{4}(\Omega)\right) \text { y } \\
& u_{m}^{\prime} \text { en un conjunto acotado de } L^{\infty}\left(0, T ; L^{2}(\Omega)\right) .
\end{aligned}
$$

Recuerde que $\|\cdot\|_{H_{0}^{1}(\Omega)}$ es una norma completa en $H_{0}^{1}(\Omega) \cap L^{4}(\Omega)$.

Usando el Teorema 2.3 de Dunford-Pettis, podemos extraer de $\left(u_{m}\right)$ una subsucesión $\left(u_{\mu}\right)$ tal que satisfagan

$$
u_{\mu} \stackrel{*}{\rightarrow} u \text { en } L^{\infty}\left(0, T ; H_{0}^{1}(\Omega) \cap L^{4}(\Omega)\right)
$$

i.e. $\int_{0}^{T}\left(u_{\mu}(t), g(t) d t \rightarrow \int_{0}^{T}(u(t), g(t)) d t, \forall g \in L^{1}\left(0, T ; H^{-1}(\Omega)+L^{\frac{4}{3}}(\Omega)\right)\right.$.

$$
u_{\mu}^{\prime} \stackrel{*}{\rightarrow} L^{\infty}\left(0, T ; L^{2}(\Omega)\right)
$$

i.e. $u_{\mu}^{\prime} \stackrel{*}{\longrightarrow} u^{\prime}$ en $D^{\prime}\left(0, T ; H_{0}^{1}(\Omega) \cap L^{4}(\Omega)\right)$.

En particular de (3.6) tenemos que $u_{m}$ está acotada en $L^{2}\left(0, T ; H_{0}^{1}(\Omega)\right.$ ) y $u_{m}^{\prime}$ está acotada en $L^{2}\left(0, T ; L^{2}(\Omega)\right)$. Por consiguiente $u_{m}$ está acotada en $H^{1}(Q):=\left\{f, f \in L^{2}\left(0, T ; H_{0}^{1}(\Omega)\right)\right.$ y $\left.f^{\prime} \in L^{2}\left(0, T ; L^{2}(\Omega)\right)\right\}$. 
Debido al Teorema 2.2 de Rellich-Kondrachoff existe una subsucesión extraída de $u_{m}$ que además de satisfacer (3.7) - (3.8), verifica:

$$
u_{\mu} \rightarrow u \text { en } L^{2}(Q) \text { fuertemente, en casi todo punto (c.t.p.). }
$$

También tenemos que $u_{m}^{3}$ pertenece al conjunto acotado de $L^{\infty}\left(0, T ; L^{\frac{4}{3}}(\Omega)\right)$

$$
u_{\mu}^{3} \stackrel{*}{\longrightarrow} w \text { en } L^{\infty}\left(0, T ; L^{\frac{4}{3}}(\Omega)\right)
$$

De (3.9), (3.10) y Lema 2.2 tenemos $w=u^{3}$ (estamos usando $U=Q, g_{\mu}=u_{\mu}^{3}$, y $s=\frac{4}{3}$ ). Usando $m=\mu$ tenemos

$$
\left\langle u_{\mu}^{\prime \prime}, w_{j}\right\rangle+a\left(u_{\mu}, w_{j}\right)=\left\langle u_{\mu}^{3}, w_{j}\right\rangle+\left(u_{\mu}^{\prime}(t), u_{\mu}^{\prime}\right)=0
$$

y como

$$
\begin{aligned}
a\left(u_{\mu}, w_{j}\right) \stackrel{*}{\longrightarrow} a\left(u, w_{j}\right) \text { en } L^{\infty}(0, T) . \\
a\left(u_{\mu}^{\prime}, w_{j}\right) \stackrel{*}{\longrightarrow}\left(u^{\prime}, w_{j}\right) \text { en } L^{\infty}(0, T) . \\
\left(u_{\mu}^{\prime \prime}, w_{j}\right) \frac{\partial}{\partial t}\left(u_{\mu}^{\prime}, w_{j}\right) \stackrel{*}{\longrightarrow}\left(u^{\prime \prime}, w_{j}\right) \text { en } D^{\prime}(0, T) . \\
a\left(u_{\mu}^{3}, w_{j}\right) \stackrel{*}{\longrightarrow}\left(u^{3}, w_{j}\right) \text { en } L^{\infty}(0, T) . \\
a\left(u_{\mu}^{\prime}, u_{\mu}^{\prime}\right) \stackrel{*}{\longrightarrow}\left(u^{\prime}, u^{\prime}\right) .
\end{aligned}
$$

entonces tenemos

$$
\frac{\partial^{2}}{\partial t^{2}}\left(u, w_{j}\right)+a\left(u, w_{j}\right)+\left(u^{3}, w_{j}\right)+\left(u_{1}, u_{t}\right)=0
$$

Por la densidad de la base $\left\{w_{1}, \ldots\right\}$ entonces

$$
\frac{\partial^{2}}{\partial t^{2}}(u, w)+a(u, w)+\left(u^{3}, w\right)+\left(u_{t}, u_{t}\right)=0, \quad \forall w \in H_{0}^{1}(\Omega) \cap L^{4}(\Omega) .
$$

Así $u$ satisface la ecuación

$$
\frac{\partial^{2}}{\partial t^{2}} u-\Delta u+u^{3}+u_{t}=0
$$

Probaremos ahora que $u(0)=u_{0}$ y $u_{t}(0)=u_{1}$. 
De (3.7), (3.8) y Lema 2.3 tenemos que $u_{\mu}(0) \rightarrow u(0)$ en $L^{2}(\Omega)$.

Por otro lado de (3.2) tenemos que $u_{\mu}(0)=u_{o \mu} \rightarrow u_{o}$ en $H_{0}^{1}(\Omega) \cap L^{4}(\Omega)$. Entonces $u(0)=u_{0}$.

De (2.1) tenemos

$$
\left(u_{\mu}^{\prime \prime}, w_{j}\right) \stackrel{*}{\longrightarrow}\left(u^{\prime \prime}, w_{j}\right) \text { en } L^{\infty}(0, T)
$$

Usando el Lema 3.2, con $X=\mathbb{R}$ tenemos

$$
\left.\left(u_{\mu}^{\prime}(0), w_{j}\right) \rightarrow\left(u^{\prime}, w_{j}\right)\right|_{t=0}=\left(u^{\prime}(0), w_{j}\right)
$$

y como $\left(u_{\mu}^{\prime}(0), w_{j}\right) \rightarrow\left(u_{1}, w_{j}\right)$ entonces $\left(u^{\prime}(0), w_{j}\right)=\left(u_{1}, w_{j}\right), \forall j$. Así $u^{\prime}(0)=u_{1}$.

\section{Prueba de la unicidad}

Sean $u, v$ soluciones de $(1.1)$ - (1.3) tales que $u(0)=v(0)=u_{0} \in H_{0}^{1}(\Omega) \cap L^{4}(\Omega)$ y $u_{t}(0)=v_{t}(0)=u_{1} \in L^{2}(\Omega)$. Defina $w:=u-v$, entonces $w$ es solución,de

$$
\begin{aligned}
& w^{\prime \prime}-\Delta w+u^{3}-v^{3}+w^{\prime}=0 \\
& w(0)=0, w_{t}(0)=0 \\
& w \in L^{\infty}\left(0, T ; H_{0}^{1}(\Omega) \cap L^{4}(\Omega)\right) \\
& w_{t}=w^{\prime} \in L^{\infty}\left(0, T ; L^{2}(\Omega)\right)
\end{aligned}
$$

Multiplicando la ecuación (3.11) por $w^{\prime}$ e integrando sobre $\Omega$ tenemos

$$
\left|w^{\prime}(t)\right|_{L^{2}}^{2}+\frac{1}{2} \frac{\partial}{\partial t}\left\{\left|w^{\prime}(t)\right|_{L^{2}}^{2}+\|w(t)\|_{H_{0}^{1}(\Omega)}^{2}\right\} \int_{\Omega}\left(v^{3}-u^{3}\right) w^{\prime} d x
$$

Observemos primero que

$$
\begin{aligned}
v^{3}-u^{3} & =(v-u)\left(u^{2}+u v+v^{2}\right) \\
& =w\left(u^{2}+u v+v^{2}\right) \\
& \leq w\left(u^{2}+\frac{1}{2}\left(u^{2}+v^{2}\right)+v^{2}\right) \\
& =w \frac{3}{2}\left(u^{2}+v^{2}\right) \\
\left|v^{3}-u^{3}\right| & \leq|w| \frac{3}{2}\left(u^{2}+v^{2}\right)
\end{aligned}
$$

También, como $u \in H_{0}^{1}(\Omega)$ y $n=3$.entonces el Teorema 2.4 de Inmersión de Sobolev (caso 1) nos garantiza que $u \in L^{6}(\Omega)$, i.e. $u^{2} \in L^{3}(\Omega)$. Obtenemos análogas conclusiones para $v$. 
Utilizando estas observaciones, la desigualdad generalizada de Hölder y que $u, v \in L^{\infty}\left(0, T ; H_{0}^{1}(\Omega)\right)$ tenemos

$$
\begin{aligned}
\left|\int_{\Omega}\left(v^{3}-u^{3}\right) w^{\prime} d x\right| & \leq \int_{\Omega}\left|v^{3}-u^{3}\right| w^{\prime} d x \\
& \leq \frac{3}{2} \int_{\Omega}|\underbrace{u^{2}+v^{2}}_{\in L^{3}}| \underbrace{w}_{\in L^{6}}|\underbrace{w^{\prime}}_{\in L^{2}}| d x \\
& \leq \frac{3}{2}\left\{\left.\left.|| u(t)\right|^{2}\right|_{L^{3}}+\left.\left.|| v(t)\right|^{2}\right|_{L^{3}}\right\}|w(t)|_{L^{6}}\left|w^{\prime}(t)\right|_{L^{2}} \\
& \leq \frac{3}{2}\left\{|u(t)|_{H_{0}^{1}}^{2}+\|v(t)\|_{H_{0}^{1}}^{2}\right\}\|w(t)\|_{H_{0}^{1}}\left|w^{\prime}(t)\right|_{L^{2}} \\
& \leq \hat{C}\left\{\|u(t)\|_{H_{0}^{1}}^{2}+\|v(t)\|_{H_{0}^{1}}^{2}\right\}\|w(t)\|_{H_{0}^{1}}\left|w^{\prime}(t)\right|_{L^{2}} \\
& \leq C\|w(t)\|_{H_{0}^{1}}\left|w^{\prime}(t)\right|_{L^{2}}
\end{aligned}
$$

Como $\left|w^{\prime}(t)\right|_{L^{2}} \geq 0$, tenemos de (3.15)

$$
\frac{1}{2} \frac{\partial}{\partial t}\left\{\left|w^{\prime}(t)\right|_{L^{2}}^{2}+\|w(t)\|_{H_{0}^{1}(\Omega)}^{2}\right\} \leq C\|w(t)\|_{H_{0}^{1}}\left|w^{\prime}(t)\right|_{L^{2}}
$$

i.e.

$$
\begin{aligned}
\frac{\partial}{\partial t}\left\{\left|w^{\prime}(t)\right|_{L^{2}}^{2}+\|w(t)\|_{H_{0}^{1}(\Omega)}^{2}\right\} & \leq 2 C\|w(t)\|_{H_{0}^{1}}\left|w^{\prime}(t)\right|_{L^{2}} \\
& \leq C\left\{\|w(t)\|_{H_{0}^{1}}^{2}+\left|w^{\prime}(t)\right|_{L^{2}}^{2}\right\},
\end{aligned}
$$

integrando de 0 a s tenemos

$$
\left|w^{\prime}(t)\right|_{L^{2}}^{2}+\|w(t)\|_{H_{0}^{1}(\Omega)}^{2} \leq c \int_{0}^{s}\left\{\|w(t)\|_{H_{0}^{1}}^{2}+\left|w^{\prime}(t)\right|_{L^{2}}^{2}\right\} d t .
$$

Usando la desigualdad de Gronwall Lema 2.1 tenemos

$$
\left|w^{\prime}(s)\right|_{L^{2}}^{2}+\|w(s)\|_{H_{0}^{1}(\Omega)}^{2}=0,
$$

de donde obtenemos $w=0$, ie. $u=v$.

Sabemos que $w_{t t} \in H^{-1}(\Omega)$ y $w_{t} \in L^{2}(\Omega)$, como no tenemos garantizado que $w_{t} \in H_{0}^{1}(\Omega)$, entonces necesitamos justificar esta conclusión previa de unicidad, para lo cual utilizaremos el Método de Visik - Ladyshenkaia, método clásico de las ecuaciones lineales hiperbólicas.

Sea $s \in(0, T]$, definimos $\psi(t)=-\int_{t}^{s} w(r) d r$, si $t \leq s$ y $\psi(t)=0$, si $t>s$. Así, $\psi$ es continua de $[0, T] \rightarrow H_{0}^{1}(\Omega), \psi(s)=0$ y $\psi^{\prime}(t)=w(t)$.

Definimos también $w_{1}(t):=\int_{0}^{t} w(r) d r$. Entonces $\psi(t)=w_{1}(t)-w_{1}(s)$ para $t \leq s$ y $\psi(0)=-w_{1}(s)$. Tomando la dualidad de la ecuación (3.11) con $\psi(t)$, e integrando de 0 a $s$ tenemos 


$$
\begin{aligned}
& \int_{0}^{s}\left(v^{3}-u^{3}, \psi\right) d t=\int_{0}^{s}\left(w^{\prime \prime}, \psi\right) d t+\int_{0}^{s} a(w, \psi) d t+\int_{0}^{s}\left(w^{\prime}, \psi\right) d t \\
& =-\int_{0}^{s}(w^{\prime}, \underbrace{\psi^{\prime}}_{=w}) d t+\int_{0}^{s} a\left(\psi^{\prime}, \psi\right) d t-\int_{0}^{s}(w, \underbrace{\psi^{\prime}}_{=w}) d t \\
& =-\frac{1}{2} \int_{0}^{s} \frac{\partial}{\partial t}|w(t)|_{L^{2}}^{2} d t+\frac{1}{2} \int_{0}^{s} \frac{\partial}{\partial t} a(\psi, \psi) d t-\int_{0}^{s}|w(t)|_{L^{2}}^{2} d t \\
& =-\int_{0}^{s}|w(t)|_{L^{2}}^{2} d t+\frac{1}{2}|w(s)|_{L^{2}}^{2}+\frac{1}{2} \underbrace{|w(0)|_{L^{2}}^{2}}_{=0}+\frac{1}{2} a \underbrace{(\psi(s)}_{=0}, \psi(s)) \\
& =-\frac{1}{2} a \underbrace{(\psi(s)}_{=w_{1}(s)}, \psi(0)) \\
& =-\int_{0}^{s}|w(t)|_{L^{2}}^{2} d t-\frac{1}{2}|w(s)|_{L^{2}}^{2}-\frac{1}{2}\left\|w_{1}(s)\right\|_{H_{0}^{1}}^{2} .
\end{aligned}
$$

Por otro lado, usando la desigualdad (3.16), la desigualdad generalizada de Hölder, la inmersión $H_{0}^{1}(\Omega) \subset L^{6}(\Omega)$ y que $u, v \in L^{\infty}\left(0, T ; H_{0}^{1}(\Omega)\right)$, tenemos

$$
\begin{aligned}
\int_{0}^{s}\left(u^{3}-v^{3}, \psi\right) d t & \leq \int_{0}^{s} \int_{\Omega}\left|v^{3}-u^{3}\right||\psi| d x d t \\
& \leq \frac{3}{2} \int_{0}^{s} \int_{\Omega} \underbrace{|w|}_{\in L^{2}} \underbrace{\left|u^{2}+v^{2}\right|}_{\in L^{3}} \underbrace{\left|w_{1}(t)-w_{1}(s)\right|}_{\in L^{6}} d x d t \\
\leq & \frac{3}{2} \int_{0}^{s}|w|_{L^{2}}\left|u^{2}+v^{2}\right|_{L^{3}}\left|w_{1}(t)-w_{1}(s)\right|_{L^{6}} d t \\
\leq & \frac{3}{2} \int_{0}^{s}|w|_{L^{2}}\left(\left|u^{2}\right|_{L^{3}}+\left|v^{2}\right|_{L^{3}}\right)\left\|w_{1}(t)-w_{1}(s)\right\|_{H_{0}^{1}} d t \\
\leq & \frac{3}{2} \int_{0}^{s}|w|_{L^{2}}\left(|u|_{L^{6}}^{2}+|v|_{L^{6}}^{2}\right)\left\|w_{1}(t)-w_{1}(s)\right\|_{H_{0}^{1}} d t \\
\leq & \frac{3}{2} \int_{0}^{s}|w|_{L^{2}}\left(\|u\|_{H_{0}^{1}}+\|v\|_{H_{0}^{1}}\right)\left\|w_{1}(t)-w_{1}(s)\right\|_{H_{0}^{1}} d t \\
\leq & C_{1} \int_{0}^{s}|w|_{L^{2}}\left\|w_{1}(t)-w_{1}(s)\right\|_{H_{0}^{1}} d t \\
\leq & C_{1} \int_{0}^{s}|w|_{L^{2}}\left\|w_{1}(t)\right\|_{H_{0}^{1}} d t+C_{1} \int_{0}^{s}|w|_{L^{2}}\left\|w_{1}(s)\right\|_{H_{0}^{1}} d t \\
\leq & \frac{C_{1}}{2} \int_{0}^{s}|w|_{L^{2}}^{2} d t+\frac{C_{1}}{2} \int_{0}^{s}\left\|w_{1}(t)\right\|_{H_{0}^{1}}^{2} d t+\frac{C_{1}}{2 \in} \int_{0}^{s}|w|_{L^{2}}^{2} d t \\
& +\frac{C_{1} \in}{2} \int_{0}^{s}\left\|w_{1}(s)\right\|_{H_{0}^{1}}^{2} d t \\
\leq & \frac{C_{1}}{2} \int_{0}^{s}|w|_{L^{2}}^{2} d t+\frac{C_{1}}{2} \int_{0}^{s}\left\|w_{1}(t)\right\|_{H_{0}^{1}}^{2} d t+\frac{C_{1}}{2 \epsilon} \int_{0}^{s}|w|_{L^{2}}^{2} d t \\
& +\frac{C_{1} \in}{2} s\left\|w_{1}(s)\right\|_{H_{0}^{1}}^{2} \\
&
\end{aligned}
$$


Entonces de la igualdad (3.17) y desigualdad (3.18) tenemos

$$
\frac{1}{2}|w(s)|_{L^{2}}^{2}+\left(\frac{1}{2}-\frac{C_{1} \in}{2} s\right)\left\|w_{1}(s)\right\|_{H_{0}^{1}}^{2} \leq C_{2} \int_{0}^{s}|w|_{L^{2}}^{2}+\left\|w_{1}(t)\right\|_{H_{0}^{1}}^{2} d t
$$

Fijando $s_{0}$, en la desigualdad (3.19) podemos escoger $\in>0$ pequeño, de modo que $\frac{1}{2}-\frac{C_{1} \epsilon}{2} s>0$ para todo $s \in\left[0, s_{0}\right]$. Luego basta escoger $\in>0$ tal que $\frac{1}{2}-\frac{C_{1} \in}{2} s_{0}>0$ y obtenemos:

$$
|w(s)|_{L^{2}}^{2}+\left\|w_{1}(s)\right\|_{H_{0}^{1}}^{2} \leq C_{3} \int_{0}^{s}|w|_{L^{2}}^{2}+\left\|w_{1}(t)\right\|_{H_{0}^{1}}^{2} d t, \quad s \in\left[0, s_{0}\right]
$$

Por otro lado como $w \in L^{\infty}\left(0, T ; H_{0}^{1}(\Omega)\right)$ y $w_{t} \in L^{\infty}\left(0, T, L^{2}(\Omega)\right)$ entonces usando el Lema 2.4 tenemos que $w \in C\left(0, T, L^{2}(\Omega)\right)$.

De la desigualdad (3.20) y usando la desigualdad de Gronwall Lema 2.1 tenemos que $w(t)=0$ en c.t.p. en $\left[0, s_{0}\right]$. Pero como $w$ es continua en $[0, T]$, entonces $w(t)=0$ para $t \in\left[0, s_{0}\right]$.

Observación $3.1 w\left(s_{0}\right)=w_{t}\left(s_{0}\right)=0$.

En efecto, ya tenemos que $w\left(s_{0}\right)=0$. Probaremos que $w_{t}\left(s_{0}\right)=0$. Observamos que $<w_{t}(r), \theta(t)>=-<w(t), \theta(t)>, \forall \theta \in \mathcal{D}\left(0, s_{0}\right)$. Entonces $\int_{0}^{s}<w_{t}(t), \theta(t)>d t=-\int_{0}^{s}<w(t), \theta^{\prime}(t)>d t$, pero $w(t)=0$ en $\left[0, s_{0}\right]$, luego, $\int_{0}^{s}<w_{t}(t), \theta(t)>d t=0, \forall \theta \in \mathcal{D}\left(0, s_{0}\right)$.

Desde que $w_{t} \in L^{\infty}\left(0, T, L^{2}(\Omega)\right)$ y $w_{t t} \in L\left(0, T, H^{-1}(\Omega)\right)$ tenemos que $w_{t} \in C\left(0, T ; H^{-1}(\Omega)\right)$ y $w_{t} \in C$ como subíndice $\left([0, T] ; L^{2}(\Omega)\right)$, luego $w_{t}\left(s_{0}\right)=0$.

Repitiendo el argumento, obtenemos el mismo resultado en $\left[s_{0}, 2 s_{0}\right], \ldots$, entonces $\forall s \in[0, T]$ tene$\operatorname{mos} w(t) \equiv 0$, i.e. $u=v$.

\section{DECAIMIENTO EXPONENCIAL}

Sabemos que

$$
\frac{\partial}{\partial t} E(t)=-\int_{\Omega}\left|u^{\prime}(t)\right|^{2} d x \leq 0,
$$

donde $E(t)=\frac{1}{2}\left\{\left|u^{\prime}(t)\right|_{L^{2}}^{2}+|\nabla u(t)|_{L^{2}}^{2}\right\}+\frac{1}{4}|u(t)|_{L^{4}}^{4}$.

Integrando la desigualdad (4.1), de 0 a $t$, tenemos que

$$
E(t)=\frac{1}{2}\left\{\left|u^{\prime}(t)\right|_{L^{2}}^{2}+|\nabla u(t)|_{L^{2}}^{2}\right\}+\frac{1}{4}|u(t)|_{L^{4}}^{4} \leq \frac{1}{2}\left\{\left|u^{\prime}(0)\right|_{L^{2}}^{2}+|\nabla u(0)|_{L^{2}}^{2}\right\}+\frac{1}{4}|u(0)|_{L^{4}}^{4}=E(0)
$$

Así, $0 \leq E(t) \leq E(0)$, i.e. está acotada y además es decreciente, debido a (4.1).

Consideremos $E(0)>0$.

Probaremos que, $\exists M>0$ tal que $\sup _{t \leq s \leq t+1} E(s) \leq M\{E(t)-E(t+1)\}, \forall t \geq 1$. 
Esto debido a Lema 2.5 de diferencias de Nakao, nos permitirá obtener: $\exists \gamma$ y $c$ constantes positivas tal que $E(t) \leq c e^{-\gamma t}$.

De (4.1) obtenemos

$$
E(t+1)-E(t)=\int_{t}^{t+1} \frac{\partial E(s)}{\partial s} d s=-\int_{t}^{t+1}\left(\int_{\Omega} u_{s}^{2} d x\right) d s,
$$

de donde tenemos

$$
E(t)-E(t+1)=\int_{t}^{t+1}\left(\int_{\Omega} u_{s}^{2} d x\right) d s \geq 0
$$

Defina $F^{2}(t)=E(t)-E(t+1)$ y $f(s):=\int_{\Omega} u_{s}^{2}(x, s) d x=\left|u_{s}(., s)\right|_{L^{2}(\Omega)}^{2} \geq 0$.

Particionamos el intervalo $[t, t+1]$ en cuatro subintervalos iguales. Usando en los intervalos extremos, el Teorema del valor medio para integrales, tenemos:

$$
\int_{t}^{t+\frac{1}{4}} f(s) d s=\frac{1}{4} f\left(t_{1}\right), t_{1} \in\left(t, t+\frac{1}{4}\right)
$$

Análogamente,

$$
\int_{t+\frac{3}{4}}^{t+\frac{1}{4}} f(s) d s=\frac{1}{4} f\left(t_{1}\right), t_{1} \in\left(t, \frac{3}{4}, t+1\right) .
$$

Así,

$$
\left|u_{t}\left(., t_{i}\right)\right|_{L^{2}(\Omega)}^{2} \leq 4 F^{2}(t) \text { para } i=1,2 .
$$

Multiplicando la ecuación (3.1) por $u$ e integrando sobre $\Omega$ tenemos

$$
\begin{aligned}
0 & =\int_{\Omega}\left(u_{t t}-\Delta u+u^{3}+u_{t}\right) u d x \\
& =\int_{\Omega} u_{t t} u d x-\int_{\Omega} \Delta u u d x+\int_{\Omega} u^{4} d x+\int_{\Omega} u_{t} u d x \\
& =\left[\frac{\partial}{\partial t}\left(\int_{\Omega} u u_{t} d x\right)-\int_{\Omega} u_{t}^{2} d x\right]+\int_{\Omega}|\nabla u|^{2} d x+\int_{\Omega} u^{4} d x+\int_{\Omega} u_{t} u d x
\end{aligned}
$$

Integrando de $t_{1}$ a $t_{2}$ la igualdad (4.3) tenemos

$$
\begin{aligned}
& \int_{t_{1}}^{t_{2}}\left(\int_{\Omega}|\nabla u(s)|^{2} d x+\int_{\Omega} u^{4}(s) d x\right) d s \\
& \quad=\int_{\Omega} u_{t t} u d x-\int_{\Omega} \Delta u u d x+\int_{\Omega} u^{4} d x+\int_{\Omega} u_{t} u d x \\
& \quad=\left[\frac{\partial}{\partial t}\left(\int_{\Omega} u u_{t} d x\right)-\int_{\Omega} u_{t}^{2} d x\right]+\int_{\Omega}|\nabla u|^{2} d x+\int_{\Omega} u^{4} d x+\int_{\Omega} u_{t} u d x
\end{aligned}
$$


Por otro lado, usando el Teorema del valor medio para integrales tenemos:

$$
\int_{t_{1}}^{t_{2}} E(s) d s=E\left(t^{*}\right)\left(t_{1}-t_{2}\right) \text {. }
$$

También tenemos,

$$
\int_{t_{1}}^{t_{2}} E(s) d s=\frac{1}{2} \int_{t_{1}}^{t_{2}} \int_{\Omega} u_{s}^{2} d x d x+\underbrace{\int_{t_{1}}^{t_{2}}\left(\frac{1}{2}|\nabla u(., s)|_{L^{2}}^{2}+\frac{1}{4} \int_{\Omega} u(x, s)^{4} d x\right) d s}_{I:=}
$$

Ahora estimaremos $I$.

Utilizando la igualdad (4.4) tenemos

$$
\begin{aligned}
& \int_{t_{1}}^{t_{2}}\left(\int_{\Omega}|\nabla u(s)|^{2} d x+\int_{\Omega} u^{2}(s) d x\right) d s \\
= & \left|\int_{\Omega} u\left(x, t_{2}\right) u_{s}\left(x, t_{2}\right) d x\right|+\left|\int_{\Omega} u\left(x, t_{1}\right) u_{s}\left(x, t_{1}\right) d x\right|+ \\
& \underbrace{\int_{t_{1}}^{t_{2}} \int_{\Omega} u_{s}^{2} d x d s}_{\leq F^{2}(t)}+\mid \underbrace{\left|\int_{t_{1}}^{t_{2}} \int_{\Omega} u(x, s) u_{s}(x, s) d x d s\right|}_{J:=} .
\end{aligned}
$$

Observe que podemos obtener las siguientes estimativas, usando la desigualdad de Hölder y desigual$\operatorname{dad}(4.2)$

$$
\left|\int_{\Omega} u\left(x, t_{i}\right) u_{s}\left(x, t_{i}\right) d x\right| \leq\left|u\left(., t_{i}\right)\right|_{L^{2}}\left|u_{t}\left(., t_{i}\right)\right|_{L^{2}} \leq 2 F(t)\left|u\left(., t_{i}\right)\right|_{L^{2}} \text { para } i=1,2 .
$$

Usando la desigualdad de Poincaré, Teorema 2.1, y sumando, tenemos

$$
\sum_{i=1}^{2}\left|\int_{\Omega} u\left(x, t_{i}\right) u_{s}\left(x, t_{i}\right) d x\right| \leq C F(t) \sup _{s \in[t, t+1]}|\nabla u(s)|_{L^{2}}
$$

Por otro lado,

$$
\begin{aligned}
J & \leq \int_{t_{1}}^{t_{2}}\left|\int_{\Omega} u(x, s) u_{s}(x, s) d x\right| d s \\
& \leq \int_{t_{1}}^{t_{2}} \sqrt{\delta}|u(., s)|_{L^{2}} \frac{1}{\sqrt{\delta}}\left|u_{s}(., s)\right|_{L^{2}} d s \\
& \leq \frac{1}{2} \int_{t_{1}}^{t_{2}}\left\{\delta|u(., s)|_{L^{2}}^{2}+\frac{1}{\delta}\left|u_{s}(., s)\right|_{L^{2}}^{2}\right\} d s \\
& \leq \frac{\delta}{2} \int_{t_{1}}^{t_{2}}|u(., s)|_{L^{2}}^{2} d s+\left\{\delta+\frac{1}{\delta}\left|u_{s}(., s)\right|_{L^{2}}^{2}\right\} d s \\
& \leq \frac{\delta}{2} \int_{t_{1}}^{t_{2}} E(s) d s \frac{1}{2 \delta} F^{2}(t)
\end{aligned}
$$

Así I queda mayorado de la siguiente forma

$$
I \leq C F(t) \sup _{s \in[t, t+1]}|\nabla u(s)|_{L^{2}}+F^{2}(t)+\frac{\delta}{2} \int_{t_{1}}^{t_{2}} E(s) d s+\frac{1}{2 \delta} F^{2}(t)
$$


donde la constante $\delta$ es arbitraria y la fijaremos en breve.

Usando la mayoración de $I$, la ecuación $(4,6)$ queda expresada

$$
\left(1-\frac{\delta}{2}\right) \int_{t_{1}}^{t_{2}} E(s) d s \leq\left(\frac{3}{2}+\frac{1}{2 \delta}\right) F^{2}(t)+C F(t) \sup _{s \in[t, t+1]}|\nabla u(s)|_{L^{2}}
$$

Tomando $0<\delta<2$, por ejemplo $\delta=1$ tenemos

$$
\begin{aligned}
\frac{1}{2} \int_{t_{1}}^{t_{2}} E(s) d s & \leq 2 F^{2}(t)+C F(t) \sup _{s \in[t, t+1]}|\nabla u(s)|_{L^{2}} \\
& \leq\left(2+c^{2} \frac{1}{\delta_{0}}\right) F^{2}(t)+\delta_{0} \sup _{s \in[t, t+1]}|\nabla u(s)|_{L^{2}} \\
& \leq\left(2+c^{2} \frac{1}{\delta_{0}}\right) F^{2}(t)+\delta_{0} \sup _{s \in[t, t+1]} E(s),
\end{aligned}
$$

donde la constante $\delta_{0}$ es arbitraria y la fijaremos en breve

Desde que $t_{2}-t_{1} \geq \frac{1}{2}$, tenemos usando (4.5) en (4.8) que

$$
E\left(t^{*}\right) \leq 4\left(2+C^{2} \frac{1}{\delta_{0}}\right) F^{2}(t)+4 \delta_{0} \sup _{s \in[t, t+1]} E(s)
$$

También tenemos que

$$
E\left(t^{*}\right) \leq E\left(t^{*}\right)+F^{2}(t) \text { para } s \in[t, t+1]
$$

Luego, de (4.9) y (4.10) tenemos

$$
\sup _{s \in[t, t+1]} E(s) \leq 4\left(\frac{9}{4}+C^{2} \frac{1}{\delta_{0}}\right) F^{2}(t)+4 \delta_{0} \sup _{s \in[t, t+1]} E(s),
$$

esto es

$$
\left(1-4 \delta_{0}\right) \sup _{s \in[t, t+1]} E(s) \leq 4\left(\frac{9}{4}+C^{2} \frac{1}{\delta_{0}}\right) F^{2}(t) .
$$

Tomando $0<\delta_{0}<\frac{1}{4}$, en particular para $\delta_{0}=\frac{1}{8}$ tenemos

$$
\sup _{s \in[t, t+1]} E(s) \leq \underbrace{8\left(\frac{9}{4}+8 C^{2}\right)}_{=\widehat{C_{0}}} F^{2}(t) .
$$

Que es lo que queríamos probar. 


\section{REFERENCIAS BIBLIOGRÁFICAS}

[1] ADAMS, R. A, Sobolev Spaces. Academic Press, New York. (1975).

[2] BREZIS H., Análisis Funcional Teoría y aplicaciones. Alianza Editorial S. A., (1984).

[3] DAFERMIS, C. M., Asymptotic behavior of solutions of evolution equations. in "Nonlinear Evolution Equations", M.G. Grandall Ed., Academic Prees, New York, pp. 103-203. (1978).

[4] LIONS, J.L, Quelques Méthodes de Résolution des Problems aux limites non lineaires. Dunod-Gauthier-Villars, Paris. (1960).

[5] LIONS, L.L. and MAGENES, E., Problemes aux limites non homogenes et applications. Vol. 1, Paris. (1968).

[6] NAKAO, M., Convergence of solutions of the wave equation with a local degenerate dissipation. Israel J. of Maths 95. pp. 25-42. (1996).

[7] NAKAO, M., Convergence of solutions of the wave equation with nonlinear dissipative term to the steady state. Memors of the Faculty of Science, Kyushu University. Ser. A., Vol. 30, No2, pp. 257-265. (1976).

[8] NAKAO, M., Decay of solutions of the wave equations with a local nonlinear dissipation. Math. Ann. 305. pp. 403-417. (1996).

[9] PAZY, A., Semigroups of Linear Operators and applications to Partial Differential Equations. Springer, New York. (1983).

[10] RENARDY, M., HRUSA, W. J. and NOHEL, J.A., Mathematical Problems in Viscoelasticity. Pirman Monographs and Surveys in Pure and Applied Mathematics 35, John Wiley \& Sons, Inc. New York. (1987).

[11] SANTIAGO, Y., Una aplicación del Lema de Nakao. Revista de los Departamentos de la Facultad de Ciencias Matemáticas, UNMSM, Nº 2 (2006). 